\title{
Stochastic cooling of electrons and positrons with EUV light
}

\author{
Alexander Zholents๑, ${ }^{*}$ Luca Rebuffi®, and Xianbo Shi॰ \\ Advanced Photon Source, Argonne National Laboratory, Argonne, Illinois 60439, USA
}

(Received 4 January 2021; accepted 11 February 2021; published 25 February 2021)

\begin{abstract}
Stochastic cooling of electrons and positrons using their extreme ultraviolet radiation is considered. A few beneficial modifications of a stochastic cooling technique are described—a petaherz-scale bandwidth, ability to apply many uncorrelated corrections to particle offsets in one cooling system, cascade amplified stochastic cooling - all resulting in a simpler and more flexible cooling system producing fast cooling without the amplifier. This approach to cooling electron and positron beams easily outperforms radiation cooling in preparing low-emittance and low-energy-spread beams in moderate-energy storage rings.
\end{abstract}

DOI: 10.1103/PhysRevAccelBeams.24.022803

\section{INTRODUCTION}

Reducing the phase-space occupied by an ensemble of identical particles and increasing their phase-space density is called cooling in beam physics [1], and it is central for many applications of beams. Cooling of electrons and positrons in storage rings occurs rather naturally due to synchrotron radiation damping [2-4]. However, synchrotron radiation is emitted in discrete quanta of light, and quantum fluctuations of the radiation counteract cooling. Therefore, the equilibrium between the radiation damping and excitation defines the final beam energy spread and emittance. Cooling by radiation is very effective at high beam energies, but not as much at energies less than a couple of hundred million electron volts. Wigglers composed of a set of alternating polarity bending magnets with a small bending radius are used to strengthen cooling at these energies $[5,6]$. They provide a dominant contribution to radiation damping and help to obtain short damping time $\tau_{S R} \sim\left(L / \rho^{2}\right)^{-1}$, where $L$ is the length of all wigglers installed in the storage ring, and $\rho$ is the bending radius. At the same time the equilibrium beam energy spread does not depend on the wiggler length and scales as $|\rho|^{-1 / 2}[6,7]$. Therefore, the beam energy spread actually increases in the wiggler-dominated storage ring-because of a much smaller $\rho$ of the wiggler magnets-than it is in any other magnet.

Stochastic cooling is a radically different beam cooling technique invented by S. van der Meer [8] and applied for cooling antiprotons in the antiproton accumulator (AA) for

\footnotetext{
*azholents@anl.gov
}

Published by the American Physical Society under the terms of the Creative Commons Attribution 4.0 International license. Further distribution of this work must maintain attribution to the author(s) and the published article's title, journal citation, and DOI. use in the $\operatorname{Sp} \bar{p} S[9,10]$. Since then it has been used in many applications involving cooling of hadron beams [11,12]. Typically, stochastic cooling of intense beams using microwave technology with several $\mathrm{GHz}$ bandwidth takes a long time, but new approaches such as optical stochastic cooling (OSC) $[13,14]$ and coherent electron cooling $(\mathrm{CeC})[15,16]$ have the potential to speed it up significantly. Moreover, it was noted in [13] that OSC can compete with radiation cooling of moderate-energy electrons, in particular when low beam energy spread is required. Indeed, it was shown in [17] that an $~ 100$ times smaller beam energy spread can be obtained in a $150-\mathrm{MeV}$ electron storage ring using OSC compared to radiation cooling. A moderate-energy cold electron beam could be used for cooling high-energy proton, antiproton, and ion beams using the electron cooling technique [18-20]. It might also be interesting to use a moderate-energy cold positron beam for cooling high-energy antiprotons, in which case relativistic antihydrogen atoms will be produced via spontaneous radiative capture of a positron by an antiproton. A proof-of-principle experiment for OSC of electrons is currently in progress at Fermilab at the moderate-energy storage ring IOTA [21,22].

In this paper we expand on the idea of using OSC for cooling electron and positron beams. We show that a drastic increase in the bandwidth of a cooling system to $\Delta f \simeq 10 \mathrm{PHz}\left(1 \mathrm{PHz}=10^{15} \mathrm{~Hz}\right)$ can be obtained by using an extreme ultraviolet (EUV) light, eliminating an optical amplifier and replacing refraction lenses with off-axis parabolic mirrors. In turn, it paves the way to a few beneficial modifications of a stochastic cooling technique.

We refer the reader to a few reviews of stochastic cooling [11,23-25] and references therein. At the core of this technique are statistical fluctuations in the distribution of beam particles in the phase-space known as shot noise [26]. According to [8], fluctuations can be revealed by slicing the phase-space and measuring slice averages, as illustrated in 


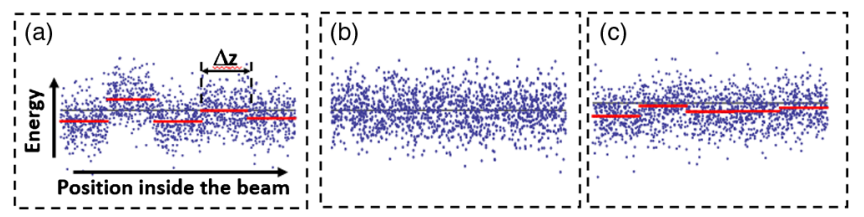

FIG. 1. An illustration of a stochastic cooling process: (a) a fragment of the beam longitudinal phase-space before correction showing exaggerated offsets of a central energy in beam slices, (b) the same fragment after correction of the offsets, and (c) the same fragment showing the appearance of newly emerged offsets after mixing of the beam particles.

Fig. 1(a). Removing these fluctuations increases the phasespace density and constitutes an elementary act of cooling, as seen in Fig. 1(b). Once that is done, particles randomly migrate between slices and new fluctuations reemerge, as seen in Fig. 1(c), at which point the beam is ready for the next elementary act of cooling. The length of the slice is $\Delta z \simeq c / \Delta f$, where $c$ is the speed of light, and the average number of slice particles is $N_{s} \simeq N \Delta z / \ell_{b}$, where $N$ is the total number of particles in the bunch, and $\ell_{b}$ is the bunch length. It is shown later that $N_{s}$ defines an ultimate cooling rate.

\section{THE METHOD}

A conventional concept of the optical stochastic cooling system is shown in Fig. 2(a). Proposed in [14] as an extension of the transit time method of stochastic cooling [27] to an optical domain, it is now adopted elsewhere [21,28-30]. However, an optical amplifier and auxiliary optics limit the bandwidth of the system. In addition, the availability of broadband optical amplifiers severely limits carrier frequency choices [28,31]. In this paper we introduce a new concept, shown in Fig. 2(b), where in addressing the problems of the original concept we remove the amplifier, replace refractive lenses with the reflective

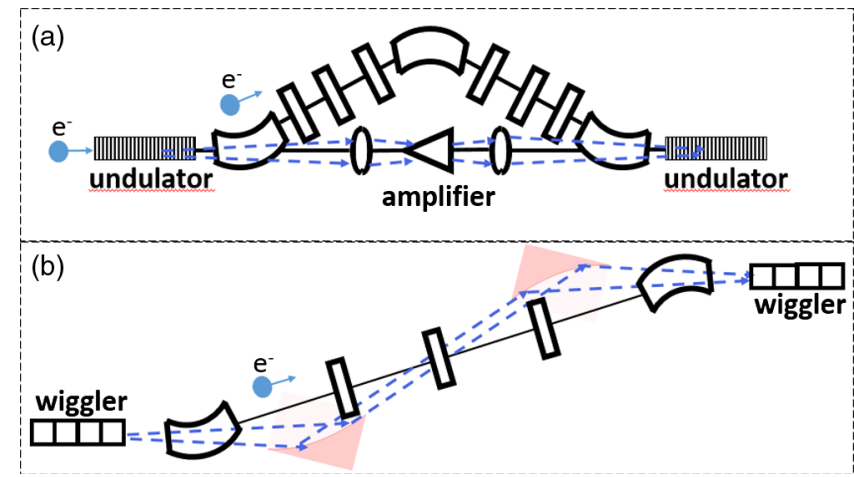

FIG. 2. (a) A conventional concept of the optical stochastic cooling system. The light radiated in the upstream undulator is focused to the optical amplifier and refocused to the downstream undulator. The electron beam follows the bypass comprised of the bending magnets and quadrupoles. (b) The new concept. optics, and replace undulators with wigglers. This concept is most suitable for cooling of electron and positron beams.

In the new concept an electron propagates the upstream wiggler (UW) and emits broadband synchrotron radiation at every bending magnet inside the wiggler. The off-axis parabolic mirror downstream of the UW collects this radiation, and the second off-axis parabolic mirror focuses it to the identical downstream wiggler (DW). The light transport has a $-I$ transport matrix, and thus the light emitted in the first magnet of the UW is focused to the first magnet of the DW, the light emitted in the second magnet of the UW is focused to the second magnet of the DW, and so on. Matching the light transport, the electron beam transport also has a $-I$ transport matrix. The entrance and exit wiggler magnets are purposely designed to provide a slight offset of the wiggling trajectory to help with a separation of the mirrors from the electron beam trajectory. The specific wiggler used later for a numerical illustration of the new concept has a period $\lambda_{W}=12.9 \mathrm{~cm}$ and a peak magnetic field of $1.6 \mathrm{~T}$. The electron with energy $\mathcal{E}_{0}=150 \mathrm{MeV}$ passes the wiggler on the trajectory shown in Fig. 3(a). We employ the wiggler with a large magnetic field to obtain an extreme ultraviolet synchrotron radiation with critical photon energy of $24 \mathrm{eV}$ in spite of using moderate-energy electrons. Following [32-34], we define the radiation field seen by an observer located on the propagation axis far from the source at a distance $R_{0}$ as

$$
E_{x_{1}}(t)=-\frac{e}{4 \pi \epsilon_{0} R_{0}} \frac{d^{2} \hat{x}}{c^{2} d t^{2}}
$$

where $e$ is the electron charge, $\epsilon_{0}$ is the dielectric constant, $\hat{x}$ is the apparent trajectory of the electron, and $t$ is the time in the observer's frame. Defining the angular acceptance of the first paraboloid mirror as $\Theta$, and using the classical electron radius $r_{e}=\frac{e^{2}}{4 \pi \epsilon_{0} m c^{2}}$, where $m$ is the electron mass at
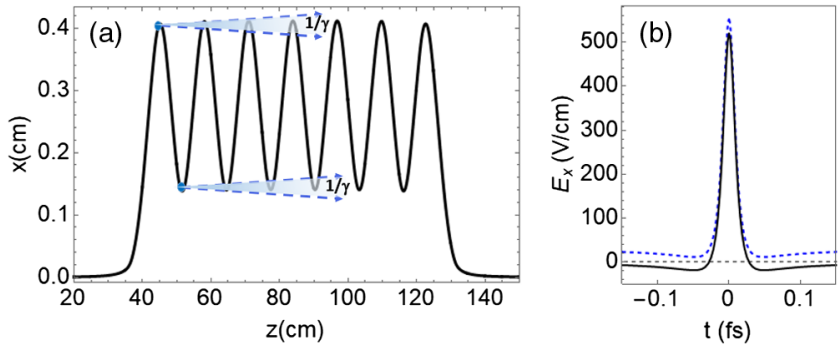

FIG. 3. (a) An electron trajectory in the wiggler with six periods; $z$ is the coordinate along the wiggler axis, and $x$ is the horizontal coordinate where trajectory oscillations take place; dashed arrows show the direction of the emitted radiation at turning points of the trajectory. (b) A pulse of electron radiation collected from one of the upstream wiggler bending magnets at a $1 / \gamma$ angle and focused to an analogous magnet in the DW; the FWHM of the pulse is 19 attoseconds and the peak field is $522 \mathrm{~V} / \mathrm{cm}$. The blue dashed curve shows the analytical result plotted with a small offset. 
rest, we write the electric field of the light transported to the DW as

$$
E_{x_{2}}(t) \simeq-\frac{m c^{2}}{e} \Theta \frac{r_{e}}{\sqrt{2 \pi} \sigma_{d}} \frac{d^{2} \hat{x}}{c^{2} d t^{2}}
$$

Here $\sigma_{d}$ is the rms vertical size of the focused light beam at a waist. It is considered to be defined by diffraction at a critical photon energy. If $\Theta>1 / \gamma$, then $1 / \gamma$ should be used in (2). The main difficulty in calculating $E_{x_{2}}$ is the calculation of $\frac{d^{2} \hat{x}}{d(t)^{2}}$. Using the prescription given in [32-34], we obtained the field plotted in Fig. 3(b). We also calculated this field using a methodology described in [35] and obtained the same result.

In each magnet of the DW, the electron interacts with the field that it radiated in the analogous magnet of the UW. As a result, as it passes every magnet it gains the energy

$$
\Delta \mathcal{E}(\tau)=e c \int E_{x_{2}}(t-\tau) \beta d t
$$

where $\beta$ is the electron horizontal velocity normalized on $c$. The exact amount of the energy gain or loss depends on the time delay, $\tau$, of the electron arrival in the DW with respect to the arrival of the light pulse, as shown in Fig. 4(a). We introduce a new variable $s=c \tau$ and approximate energy kick by the function $\Delta \mathcal{E}(s)=\tilde{A} f\left(s / s_{0}\right)$, where $f(x)=\exp \left(-(x-1)^{2} / 2\right)-\exp \left(-(x+1)^{2} / 2\right)$, and $\tilde{A}$ is the dimensional constant. $|\Delta \mathcal{E}(s)|$ has a maximum at $s= \pm s_{0}$. The width of $\Delta \mathcal{E}(s)$ at the base is $\sim 7 s_{0}$. Since the radiation fields produced by electrons outside of this range do not impact electrons inside this range, the slice length $\Delta z \simeq 7 s_{0}=25 \mathrm{~nm}$.

It is required in the transient time method of stochastic cooling $[14,27]$ employed in our study that an electron with equilibrium energy and without offset from the beam orbit
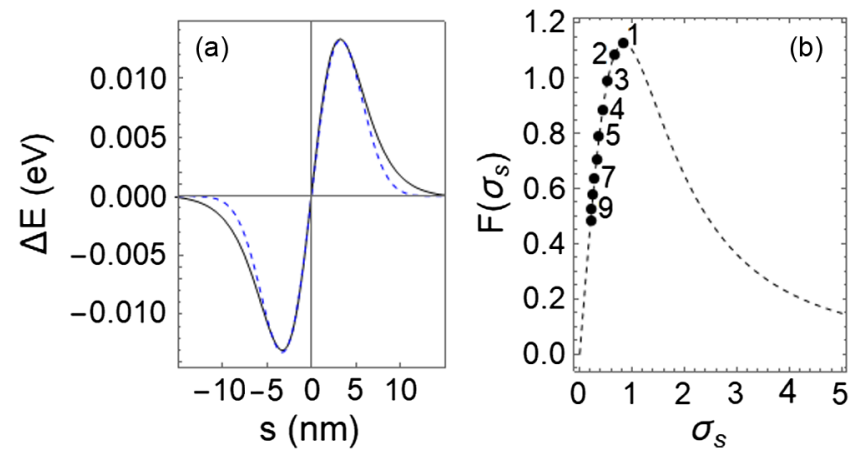

FIG. 4. (a) The energy change of the electrons calculated using (3) (black curve) and an approximating function (dashed blue curve). (b) The normalized cooling force. Only one half of the electrons with positive values is shown. The dots show optimal $\sigma_{s 0}$ for the cascade with the number of cooling sections written next to the dot. experiences no energy change passing the cooling system. This imposes several important constraints on the design of the electron and light transport between the wigglers. Let $\Delta t_{e}$ be the time it takes for the equilibrium electron to pass the trajectory from the UW to the DW and $\Delta t_{l}$ be the time it takes for its radiation to propagate from the UW to the DW. The difference between these times is constrained to produce a delay $\Delta t=\Delta t_{l}-\Delta t_{e}$ and to achieve a condition where the equilibrium electron propagates the magnet without energy change in the interaction with its own radiation, in which case it "meets" the peak of the radiation pulse at the peak of the trajectory where the horizontal velocity of the electron is zero. This is a unique delay, and it is used to define $s=0$ in Fig. 4(a). Subsequently, the equilibrium electron arrives at the second magnet of the DW ahead of the light pulse it emitted at the second magnet of the UW with the same delay $\Delta t$. Thus, the light interaction with the electron there does not change the electron energy. This is repeated at every wiggler magnet, and the net energy change of the equilibrium electron over the entire wiggler is zero. Once $\Delta t$ has been set up, it has to be maintained by a feedback system with an ultrahigh precision on the order of tens of attoseconds. An example of the feedback system is described in [36], where a glass wedge is considered for a correction of light arrival time. Operating in EUV will likely require the feedback to act on the electron beam trajectory.

Meanwhile, electrons with energy offsets from the equilibrium follow different trajectories and arrive at the DW having $s \neq 0$. The time-of-flight properties of the electron transport system are used to control $s$ and facilitate corrections of energy offsets in their interactions with the radiation fields emitted in the UW, as illustrated in Fig. 4(a). Electrons with energies higher than the equilibrium energy are decelerated, giving part of their excess energy to the field, and the electrons with energies smaller than the equilibrium energy are accelerated by taking part of the field energy. This constitutes an elementary act of cooling as it reduces the beam energy spread. The electron's transverse offsets from equilibrium can also be corrected using the time-of-flight properties of the electron transport system and introducing a small dispersion function at the locations of the UW and DW, as proposed in [14] in an analogy to Palmer's method of stochastic cooling [37]. For clarity of the presentation, we begin with an analysis of the beam energy spread cooling and later explain what has to be changed to obtain simultaneous cooling of the beam energy spread and the beam emittance.

\section{A. One-dimensional cooling}

We use the symbol $\delta=\delta \mathcal{E} / \mathcal{E}_{0}$ for the electron relative energy offset from the equilibrium energy, $\mathcal{E}_{0}$, and consider a Gaussian distribution $\left(\sqrt{2 \pi} \sigma_{\delta}\right)^{-1} e^{-\delta^{2} / 2 \sigma_{\delta}^{2}}$ in the electron beam with the rms value $\sigma_{\delta}$. Let us now consider the design of an electron transport between the wigglers that has only 
one nonzero time-of-flight parameter $R_{56}$, such as $s=R_{56} \delta$, and obtain the energy change of one electron after propagation of one magnet in the DW, accounting for all $N_{s}$ electrons within the same beam slice

$$
\delta_{i c}=\delta_{i}-A f\left(R_{56} \delta_{i} / s_{0}\right)-A \sum_{k \neq i}^{N_{s}} f\left(R_{56} \delta_{k} / s_{0}\right) .
$$

Here $\delta_{i}$ and $\delta_{i c}$ are the electron energy offsets before and after correction, respectively, and $A=\tilde{A} / \mathcal{E}_{0}$. The second term on the right side of (4) is the mathematical expression for the energy correction described above, i.e., the result of the electron interaction with its own field radiated in the UW. The third term is the result of the electron interaction with the fields radiated in the UW by other electrons within the same slice. This term represents a random distortion to $\delta$. Although a single electron is inherently a quantum object, we note that a pure classical calculation (4) is justified according to [38] when the electron is expected to experience many thousands of corrections for its energy offset during the damping time.

One important feature of the wiggler design is that the bending angle $\phi$ of each wiggler magnet and $\lambda_{W}$ are selected to yield the condition

$$
\frac{\lambda_{W}}{4 \pi^{2} \Delta z} \phi^{2} \sigma_{\delta} \geq 1
$$

in which the electrons with different energies have enough time to migrate between neighboring slices while the beam passes one wiggler magnet. Therefore, each electron moving from one wiggler magnet to the next appears to be surrounded by a fully refreshed subset of other electrons. Subsequently, in every DW magnet each electron interacts with a fully refreshed subset of the fields radiated by other electrons in the UW. As a result, each electron accumulates correction actions represented by the second term in (4) at every wiggler magnet while the distortion represented by the third term in (4) fluctuates. In other words, we change the order of the actions shown in Fig. 1. Departing from classical stochastic cooling, we collect a signal from the first UW magnet, defer the correction action, and proceed with mixing electrons between slices and collecting the signal from the second magnet, and so on. Once the collection of signals from all UW magnets is complete, we proceed with correction actions in the DW, applying them one by one in the same sequence as the signals in the UW. Using (4) and averaging over the electron energy distribution, we obtain the change of the rms beam energy in each magnet of the DW, $\Delta \delta^{2}=\overline{\delta_{i c}^{2}-\delta_{i}^{2}}$ as

$$
\Delta \delta^{2}=-\frac{2 A}{\sqrt{2 \pi} \sigma_{\delta}} \int_{-\infty}^{\infty} \delta e^{-\frac{\delta^{2}}{2 \sigma_{\delta}^{2}}} f\left(\frac{R_{56} \delta}{s_{0}}\right) d \delta+A^{2} N_{s} \overline{\left(\frac{R_{56} \delta}{s_{0}}\right)^{2}},
$$

and define $\alpha_{\delta}=-n_{W} \Delta \delta^{2} / \sigma_{\delta}^{2}$, where $n_{W}$ is the total number of bending magnets in the wiggler, as the cooling parameter characterizing a single electron beam pass through a cooling system shown in Fig. 2(b). The first term in (6) defines cooling of electrons by their own fields radiated in the UW, and the second term defines "heating" of electrons by fields radiated by other slice electrons. Performing integration and analyzing the result, we find that $\alpha_{\delta}$ is at a maximum when

$$
A \cdot N_{s} \simeq \frac{2 \pi s_{0} \sigma_{s}^{2} e^{-\frac{1}{2+2 \sigma_{s}^{2}}}}{R_{56}\left(1+\sigma_{s}^{2}\right)^{3 / 2}}
$$

in which case

$$
\alpha_{\delta \max } \cdot N_{s} \simeq n_{W} \frac{4 \pi \sigma_{s}^{2} e^{-\frac{1}{1+\sigma_{s}^{2}}}}{\left(1+\sigma_{s}^{2}\right)^{3}}
$$

where a new variable $\sigma_{s}=R_{56} \sigma_{\delta} / s_{0}$ is used for brevity. The right side of (8) has the maximum $\alpha_{\delta \max } \cdot N_{s} \simeq n_{W}$ at $\sigma_{s}=\sigma_{s 0}=0.84$. Therefore, obtaining the fastest cooling rate requires an electron transport system with $R_{56}=0.84 s_{0} / \sigma_{\delta}$, in which case

$$
\alpha_{\delta \max } \simeq n_{W} / N_{s} \quad \text { and } \quad A \cdot N_{s} \simeq 1.76 \sigma_{\delta},
$$

and $R_{56}$ has to increase dynamically if $\sigma_{\delta}$ decreases by cooling, to keep $\sigma_{s 0}=0.84$. It is worth mentioning that operating in the EUV facilitates and makes easier the transverse slicing introduced in $[14,39]$ to further reduce $N_{s}$.

In the steady state case when cooling simply balances the excitation, $R_{56}$ remains unchanged. We notice that when $A=\Delta E\left(s_{0}\right) / \mathcal{E}_{0} \simeq 10^{-10}$ and $\sigma_{\delta} \simeq 2 \times 10^{-4}$, as in the example considered in this paper, the $N_{s}=3.5 \times 10^{6}$ according to (9). It corresponds to a 6.8-kA peak current since $\Delta z / c$ is $\sim 80$ attoseconds. Obviously, using this beam is not possible for many reasons. Using a less intense beam and smaller $N_{s}$ does not help to obtain faster cooling if $A$ remains the same, but then the second term in (6), $\sim A^{2} N_{s}$, can be ignored, and thus the cooling parameter becomes

$\alpha_{\delta}=n_{W} \frac{A}{\sigma_{\delta}} \frac{4 \sigma_{s} e^{-\frac{1}{2+2 \sigma_{s}^{2}}}}{\left(1+\sigma_{s}^{2}\right)^{3 / 2}} \simeq 1.125 n_{W} \frac{A}{\sigma_{\delta}}\left(\right.$ at $\left.\sigma_{s}=\sigma_{s 0}\right)$.

This result has a simple interpretation-a small part of the electron beam energy spread is taken away by each single act of cooling. The rate of cooling is defined by a number of these acts per second, and the cooling time is defined by the time it takes to accumulate the sum of subtractions equal to $\sigma_{\delta}$. To increase the rate of cooling and reduce the cooling time, we propose using in one storage ring several identical cooling systems assembled back-to-back, as shown in Fig. 5, in which case the DW of the upstream cooling system automatically becomes the UW of the 


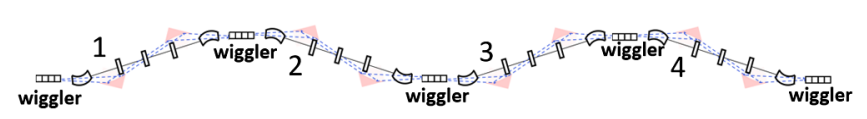

FIG. 5. A schematic of four cooling sections assembled backto-back to obtain cascade amplified stochastic cooling.

following cooling system. We call this approach cascade amplified stochastic cooling. Indeed, the electron radiation originated in every wiggler is now cascaded downstream to facilitate the interaction of electrons with this light in all remaining downstream wigglers. With this arrangement the cooling parameter gets a boost that depends on a number of cooling systems in the cascade $n_{s}$,

$\alpha_{\delta}\left(n_{s}\right)=n_{W} \frac{A}{\sigma_{\delta}} \sum_{i=0}^{n_{s}-1}\left(n_{s}-i\right) F\left((i+1) \frac{R_{56}\left(n_{s}\right) \sigma_{\delta}}{s_{0}}\right)$,

where $F(x)=4 x e^{-\frac{1}{2+2 x^{2}}}\left(1+x^{2}\right)^{-3 / 2}$. This function is shown in Fig. 4(b) and has been already used in (10). Now the optimal value of $R_{56}$ that maximizes $\alpha\left(n_{s}\right)$ depends on $n_{s}$,

$$
R_{56}\left(n_{s}\right)=\frac{s_{0}}{\sigma_{\delta}}\left(\frac{1.18}{\sqrt{n_{s}}}-0.16\right), \quad n_{s} \geq 2 .
$$

This is because the time-of-flight delays of the electrons keep increasing with every new downstream cooling system. Therefore, $\sigma_{s 0}$ has to be set to a smaller value to facilitate the most efficient exposure of the electrons to a damping force, as shown in Fig. 4(b). Obviously, the action of a single cooling system becomes weaker, but the repetitive use of light in the cascade easily overcomes this loss:

$$
\alpha_{\delta}\left(n_{s}\right) \simeq \frac{A}{\sigma_{\delta}} n_{W}\left(0.74 n_{s}+0.41 n_{s}^{2}\right) .
$$

When deriving (13) we neglected a small attenuation of the light in a transport. We also note that the field radiated by each electron in the UWs increases in some cases and decreases in other cases in the interactions with that electron in the DWs. However, it does not change the above result since cooling takes many thousands of electron passes through the cascade, and the impact of these variations averages to zero over this time.

\section{B. Two-dimensional cooling}

Adding cooling of the horizontal emittance is conceptually simple. What is required is to make the time-offlight delay $s$ be dependent on both transverse and energy offsets of the electrons from the equilibrium, i.e., $s=R_{51} x+R_{52} x^{\prime}+\left(R_{56}+R_{51} \eta+R_{52} \eta^{\prime}\right) \delta$, where $x$ and $x^{\prime}$ are horizontal offsets and angles of electrons at the beginning of the cooling system due to the beam emittance,
$R_{51}$ and $R_{52}$ are two new time-of-flight parameters of the cooling system, and $\eta$ and $\eta^{\prime}$ are the dispersion function and its derivative at the beginning of the cooling system. Although there are many combinations of these parameters producing simultaneous cooling of the energy spread and the emittance, the most transparent one in the case of the $-I$ transport matrix for the cooling system is when $R_{52}=0$ and $\eta^{\prime}=0$. The brevity of formulas is also assisted by a choice of the storage ring lattice with horizontal Twiss functions at the beginning of cooling system $\beta_{x}$ and $\alpha_{x}=0$. Obtaining two-dimensional (2D) cooling parameters $\alpha_{\delta 2 D}$ (for the energy spread) and $\alpha_{x 2 D}$ (for the emittance) in this case is similar to one-dimensional (1D) analysis. The only difference is that in addition to averaging over the energy distribution, as in (6), we need averaging over the distribution in $x$ that is assumed to be $\left(\sqrt{2 \pi} \sigma_{x}\right)^{-1} e^{-\frac{x^{2}}{2 \sigma_{x}^{2}}}$, where $\sigma_{x}$ is the rms horizontal beam size at the beginning of the cooling system. Skipping some mathematics, we write the result as

$$
\begin{aligned}
& \alpha_{\delta 2 D}=n_{W} A \frac{R_{56}-R_{51} \eta}{s_{0}} \frac{4 e^{-\frac{1}{2+2\left(\sigma_{s 2 D}\right)^{2}}}}{\left(1+\left(\sigma_{s 2 D}\right)^{2}\right)^{3 / 2}} \\
& \alpha_{x 2 D}=n_{W} A \frac{R_{51} \eta}{s_{0}} \frac{4 e^{-\frac{1}{2+2\left(\sigma_{s 2 D}\right)^{2}}}}{\left(1+\left(\sigma_{s 2 D}\right)^{2}\right)^{3 / 2}},
\end{aligned}
$$

where $\sigma_{s 2 D}=\sqrt{R_{51}^{2} \sigma_{x}^{2}+\left(R_{56}-R_{51} \eta\right)^{2} \sigma_{\delta}^{2}} / s_{0}$. For one specific example we further assume that $\eta$ is selected to yield $\sigma_{x}=\eta \sigma_{\delta}$ and $R_{51}$ is selected to yield $R_{51} \eta=\frac{1}{2} R_{56}$ and obtain

$$
\alpha_{x 2 D}=\alpha_{\delta 2 D}=n_{W} \frac{1}{\sqrt{2}} \frac{A}{\sigma_{\delta}} \frac{4\left(\sigma_{s 2 D}\right) e^{-\frac{1}{2+2\left(\sigma_{s 2 D}\right)^{2}}}}{\left(1+\left(\sigma_{s 2 D}\right)^{2}\right)^{3 / 2}} .
$$

Analogous to 1D cooling, both cooling parameters attain maximum values when $\sigma_{s 2 D}=0.84$, in which case $R_{56}=\sqrt{2} \frac{0.84 s_{0}}{\sigma_{\delta}}$ and $R_{51}=\frac{1}{\sqrt{2}} \frac{0.84 s_{0}}{\sigma_{x}}$. The 2D cooling parameters in (15) are smaller than the 1D cooling parameter in (10) by a factor $1 / \sqrt{2}$. Many choices with unequal $\alpha_{x 2 D}$ and $\alpha_{\delta 2 D}$ values are possible, including one giving $\alpha_{\delta 2 D}=0$ and $\alpha_{x 2 D}=\alpha_{\delta}$, where $\alpha_{\delta}$ is the previously defined 1D cooling parameter. It can be achieved with selection of $R_{51} \eta=R_{56}$. This result complements (10) and verifies the generalization of $1 \mathrm{D}$ cooling to 2D cooling given in (14).

Finally, we note that arranging several cooling systems into a cascade in the case of the $2 \mathrm{D}$ cooling requires selecting the opposite signs for $R_{51}$ in odd and even cooling systems within the cascade. Evidently, selection of the absolute value of $R_{51}$ depends on the number of cooling systems $n_{s}$ and has to follow the rule given in (12) for $R_{56}$.

In the Appendix we consider an illustrative design of a racetrack-like storage ring with a circumference $C \simeq 300 \mathrm{~m}$ 
and two 125-m-long straight sections. Each straight section contains a cooling cascade with $n_{s}=7$ and wigglers with $n_{W}=20$. Using (13) with $A=8.7 \times 10^{-11}$ and $\sigma_{\delta}=2 \times 10^{-4}$, we calculate the cascade's cooling parameter $\alpha_{\delta 2 D}\left(n_{s}\right)=2.2 \times 10^{-4}$ and the stochastic cooling damping time in the ring with two cascades $\tau_{S C}=C /\left(c \cdot 2 \alpha_{\delta 2 D}\right)=$ $2 \mathrm{~ms}$. The calculated synchrotron radiation damping time in this ring is $\tau_{S R}=31 \mathrm{~ms}$, and the relative energy spread without stochastic cooling is $2 \times 10^{-4}$. Hypothetically, $\tau_{S R}=3 \mathrm{~ms}$ can be obtained by packing $250 \mathrm{~m}$ of two straight sections entirely with wigglers.

\section{SUMMARY}

In summary, OSC in EUV offers several remarkable advantages such as wide bandwidth, the ability to apply many uncorrelated corrections to particle offsets in one cooling system, the ability to combine several cooling systems into a highly efficient cooling cascade, and the ability to cool high intensity beams at practically the same rate as low intensity beams. Moreover, the cooling system becomes much simpler and more flexible without the amplifier that conventional OSC relies on. Indeed, the cooling system can operate without changes in a wide range of beam energies, while the beam energy is bounded to a narrow range limited by the amplifier bandwidth and tunability of the undulators in conventional OSC. On the downside of the transition to EUV is increased sensitivity to stability of the time-of-flight property of the cooling system.

\section{ACKNOWLEDGMENTS}

A. Z. is grateful to Maofei Qian from ANL for providing a magnetic design of the wiggler and Valery Lebedev from Fermilab for careful reading of the manuscript and valuable suggestions for improvement of the presentation. This paper is based upon work supported by Laboratory Directed Research and Development (LDRD) funding from Argonne National Laboratory, provided by the Director, Office of Science, Office of Basic Energy Sciences, of the U.S. Department of Energy under Contract No. DE-AC0206CH11357.

\section{APPENDIX: STORAGE RING AND COOLING SYSTEM DESIGN}

Figure 6 shows a schematic of the cooling system and Fig. 7 shows Twiss functions and a dispersion function. The lattice design of the cooling system is very simple. The quadrupoles make a FODO channel with the horizontal betatron phase advance per cell $\phi_{x}=\pi / 2$ and with the vertical betatron phase advance $\phi_{y}=\pi / 4$ in all internal cells and $\phi_{y}=\pi / 2$ in the entrance and exit cells. Therefore, the horizontal phase advance between the magnets is $\pi$, in which case the dispersion function excited by the first magnet M1 is canceled by the second magnet M2, and the

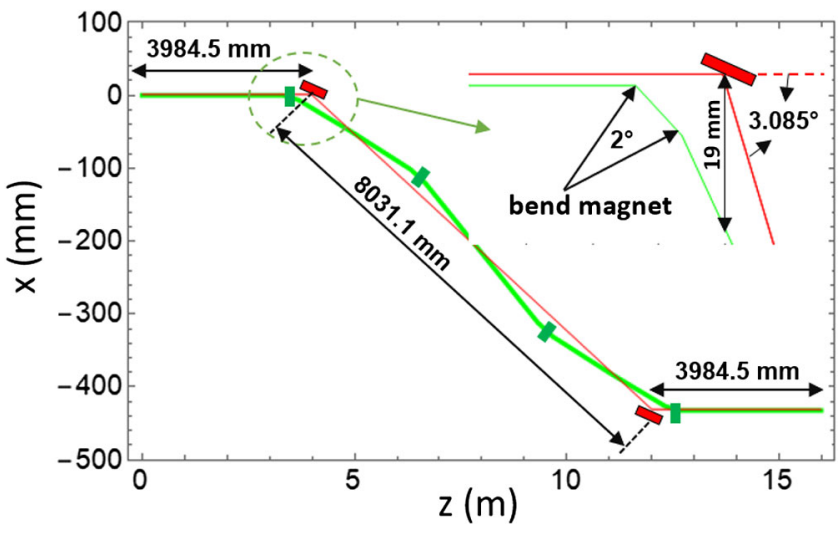

FIG. 6. A schematic of the cooling system. The red line shows the light path, and the two small red boxes are the off-axis parabolic mirrors. The green line shows the electron path, and the four green boxes are bending magnets. The quadrupole lenses used in the electron beamline are not shown. The inset shows the details of the mirror separation from the electron beam trajectory. A small initial offset of the light trajectory seen there is due to the 1.4-mm electron beam orbit offset in the wiggler (see Fig. 3).

dispersion function excited by the third magnet M3 is canceled by the fourth magnet M4. The horizontal (vertical) betatron phase advance over the entire cooling system from the entrance to the exit is $5 \pi(3 \pi)$, and the entire transport matrix is $-I$ in both planes. Separated by a unit transport matrix, the trim magnets TM1 and TM2 are used to adjust $R_{56}$ and $R_{51}$. TM1 excites a small dispersion bump that introduces a small additional dispersion with one sign in M2 and with the opposite sign in M3. TM2 has the opposite polarity to TM1 and cancels the bump. The electron beam trajectory is guided by magnets M1, M2, M3, and M4. It is carefully selected to (a) match the light beam trajectory guided by two parabolic mirrors, and (b) match the time it takes for the equilibrium electron to propagate from the UW to the DW to the time it takes for a light radiated

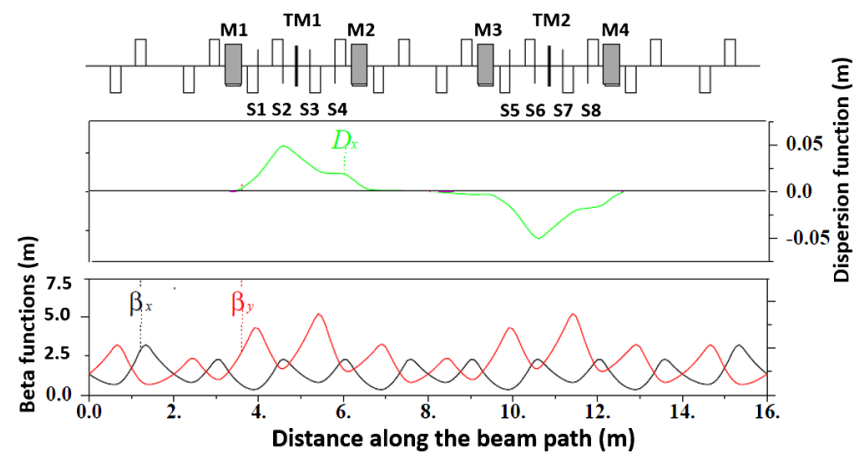

FIG. 7. This plot shows horizontal $\beta_{x}$ and vertical $\beta_{y}$ Twiss functions in the bottom panel, dispersion function $D_{x}$ in the middle panel, and a sequence of magnetic elements at the top. The open rectangles are the quadrupoles, the grey rectangles are bending magnets, and the two narrow black rectangles are trim magnets. 


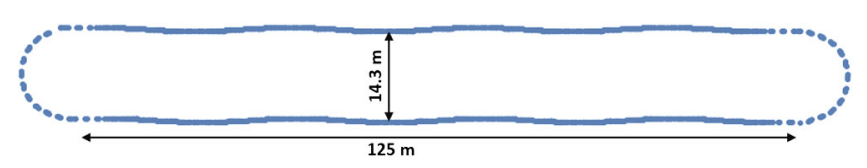

FIG. 8. The layout of the storage ring.

by that electron in the UW to propagate to the DW. Each magnet is $0.35 \mathrm{~m}$ long and bends the trajectory by $2^{\circ}$. As seen in Fig. 6, the direction of the magnetic field in M3 and M4 is opposite to that in M1 and M2. Four pairs of sextupoles-(S1:S5), (S2:S6), (S3:S7), and (S4:S8)-are used to zero second-order contributions to the time-offlight of the electron coming from the electron transverse and energy offsets from the equilibrium. The sextupoles in each pair have opposite polarities and are located in identical places inside the respective cells having the same Twiss functions. The horizontal (vertical) betatron phase advance between the sextupoles is $2 \pi(\pi)$. Therefore, the geometrical aberrations introduced by the first sextupole in the pair are canceled by the second sextupole. With $1 / \gamma$ angular acceptance, the mirror's vertical acceptance is $13.6 \mathrm{~mm}$, and the mirror's horizontal acceptance is $16.3 \mathrm{~mm}$, which also accommodates a $2.7-\mathrm{mm}$ separation of the light source points that belong to the wiggler magnets with the positive and negative polarities of the magnetic field, as seen in Fig. 3. Therefore, with a $3.085^{\circ}$ angle of grazing incidence of the light to the mirror, the mirror length is $303 \mathrm{~mm}$, and the light reflection efficiency is close $100 \%$. The mirror's focal length is $3.984 \mathrm{~m}$.

We arrange seven cooling systems into a 112-m-long cooling cascade by stacking them together back-to-back and alternating the polarities of all the magnets and sextupoles in odd and even cooling systems within the cascade. Two such cascades are used in a racetrack-like storage ring shown in Fig. 8. The circumference of the ring is $297.3 \mathrm{~m}$, and the length of the straight section is $125 \mathrm{~m}$. Each $180^{\circ}$ arc has four identical cells containing two $1.3-\mathrm{m}-$ long magnets with an $18^{\circ}$ bending angle plus one focusing and one defocusing quadrupole. An extra cell with only one bending magnet is used at each end of the arc for dispersion suppression. The synchrotron radiation damping in this ring at a beam energy of $150 \mathrm{MeV}$ is dominated by 16 wigglers that are used in two cooling cascades. The wiggler period is $12.9 \mathrm{~cm}$, and the peak magnetic field is $1.6 \mathrm{~T}$. Each wiggler contains 10 periods and, thus, $n_{W}=20$. The damping time is equal to $31 \mathrm{~ms}$. Quantum fluctuations of the wiggler's synchrotron radiation is mostly responsible for excitation of the beam energy spread in a low-intensity beam; on average they add $\sim 1 \mathrm{eV}$ to the electron beam energy spread after one orbit turn. A balance between the radiative excitation and cooling produces an rms relative energy spread in the low intensity beam equal to $2 \times 10^{-4}$.

Calculations show that stochastic cooling in the same ring using two cooling cascades with $n_{s}=7$ is capable of removing on average $\sim 13 \mathrm{eV}$ from the electron beam energy spread in one orbit turn. This rate of cooling is 13 times faster than the growth rate due to quantum fluctuations. However, the intrabeam scattering (IBS) in the low- and medium-energy electron and positron storage rings typically causes larger excitation of the beam energy spread than quantum fluctuations. Furthermore, the IBS rate depends on the electron beam peak current. Thus, it is interesting to find the peak current when the IBS rate matches the rate of stochastic cooling. We used the computer code MAD [40] to calculate the peak current and obtained $0.65 \mathrm{~A}$. In this calculation we used a horizontal geometrical emittance of $9.6 \mathrm{~nm}$, a vertical geometrical emittance of $1.5 \mathrm{~nm}$, and a relative energy spread of $2 \times 10^{-4}$. The specific reason for selecting these beam parameters is that such a beam could be of interest to cooling of protons in a future electron ion collider (EIC) [41], although the peak current might be insufficient. Therefore, we note that the peak current can be increased proportional to the increase of the electron beam energy spread. The final comment is that obtaining an electron beam with the same parameters as listed above in the same electron ring is not possible with radiation cooling.

[1] A. Sessler, Comment on the word "cooling" as it is used in beam physics, AIP Conf. Proc. 821, 3 (2006).

[2] A. Kolomenski and A. Lebedev, The effect of radiation on the motion of relativistic electrons in synchrotrons, in Proc. CERN Symposium (CERN, Geneva, 1956), p. 447.

[3] K. W. Robinson, Radiation effects in circular electron accelerators, Phys. Rev. 111, 373 (1958).

[4] M. Sands, The physics of electron storage rings, Tech. Report No. SLAC 121, 1970.

[5] D. Cline, A. Garren, H. Herr, F. E. Mills, C. Rubbia, A. Ruggiero, and D. Young, High energy electron cooling to improve the luminosity and lifetime in colliding beam machines, in IEEE Transaction on Nuclear Science, Vol. NS-26(1979), pp. 3472-3475.

[6] M. Gentner, R. Brinkmann, Y. Derbenev, D. Husmann, and C. Steier, On the possibilities of electron cooling for HERA, Nucl. Instrum. Methods Phys. Res., Sect. A 424, 277 (1999).

[7] Handbook of Accelerator Physics and Engineering, 2nd ed., edited by A. Chao, M. Tigner, F. Zimmermann, and K.-H. Mess (World Scientific Publishing, Singapore, 2013), Chap. 3.1.4.5.

[8] S. van der Meer, Stochastic damping of betatron oscillations, CERN Report No. CERN ISR-PO 72/31, 1973.

[9] S. van der Meer, Stochastic cooling and the accumulation of antiprotons, Rev. Mod. Phys. 57, 689 (1985).

[10] H. Koziol and D. Möhl, The CERN antiproton collider programme: Accelerators and accumulation rings, Phys. Rep. 403-404, 91 (2004).

[11] J. Marriner, Stochastic cooling overview, Nucl. Instrum. Methods Phys. Res., Sect. A 532, 11 (2004). 
[12] M. Blaskiewicz and J. M. Brennan, Bunched beam stochastic cooling in a collider, Phys. Rev. Accel. Beams 10, 061001 (2007).

[13] A. A. Mikhailichenko and M.S. Zolotorev, Optical Stochastic Cooling, Phys. Rev. Lett. 71, 4146 (1993).

[14] M. S. Zolotorev and A. A. Zholents, Transit-time method of optical stochastic cooling, Phys. Rev. E 50, 3087 (1994).

[15] Y. Derbenev, Use of an electron beam for stochastic cooling, in Proc. Int. Workshop on Beam Cooling and Related Topics (Cool07), Report No. JLAB-ACP-07-700, 2007.

[16] V. N. Litvinenko and Y. S. Derbenev, Coherent Electron Cooling, Phys. Rev. Lett. 102, 114801 (2009).

[17] A. A. Zholents and M.S. Zolotorev, A proposal for the generation of ultra-short x-ray pulses, Nucl. Instrum. Methods Phys. Res., Sect. A 358, 455 (1995).

[18] G. Budker, An effective method of damping particle oscillations in proton and antiproton storage rings, At. Energ. 22, 346 (1967).

[19] V. V. Parkhomchuk and A. N. Skrinsky, Electron cooling: 35 years of development, Phys. Usp. 43, 433 (2000).

[20] H. Poth, Electron cooling: Theory, experiment, application, Phys. Rep. 196, 135 (1990).

[21] J. Jarvis, S. Chattopadhyay, V. Lebedev, H. Piekarz, P. Piot, A. L. Romanov, and J. Ruan, Optical stochastic cooling program at Fermilab's integrable optics test accelerator, in Proc. North American Particle Accelerator Conference (NAPAC2019), Lansing, Michigan (2019), https://frib.msu .edu/events/2019/napac19/.

[22] V. Lebedev, Optical stochastic cooling, ICFA Beam Dyn. Newslett. 65, 100 (2014).

[23] S. Chattopadhyay, On stochastic cooling of bunched beams from fluctuation and kinetic theory, Thesis Univ. of Berkeley, California, Tech. Rep. LBL-14826, 1982.

[24] D. Möhl, Stochastic cooling for beginners, Conf. Proc. C 831011, 97 (1983).

[25] F. Caspers, Techniques of stochastic cooling, in Workshop on Beam Cooling and Related Topics, Report No. CERNPS-2001-017-RF, 2001.

[26] W. Schottky, Über spontane stromschwankungen in verschiedenen elektrizitätsleitern, Ann. Phys. (Berlin) 362, 541 (1918).

[27] W. Kells, New approach to stochastic momentum cooling", in 11th International Conference on High-Energy Accelerators, Geneva, Switzerland (1980), p. 777.
[28] M. Babzien, I. Ben-Zvi, I. Pavlishin, I. V. Pogorelsky, V. E. Yakimenko, A. A. Zholents, and M. S. Zolotorev, Optical stochastic cooling for RHIC using optical parametric amplification, Phys. Rev. Accel. Beams 7, 012801 (2004).

[29] W. Franklin, Optical stochastic cooling proof-of-principle experiment, in Proc. Particle Accelerator Conference (PAC2007), Albuquerque, New Mexico (IEEE, New York, 2007), pp. 1904-1906.

[30] M. B. Andorf, W. F. Bergan, I. V. Bazarov, J. M. Maxson, V. Khachatryan, D. L. Rubin, and S. T. Wang, Optical stochastic cooling with an arc bypass in the Cornell Electron Storage ring, Phys. Rev. Accel. Beams 23, 102801 (2020).

[31] A. Zholents and M. Zolotorev, An amplifier for optical stochastic cooling, in Proc. Particle Accelerator Conference (PAC 97), Vancouver, British Columbia, Canada (IEEE, New York, 1997), p. 1804.

[32] R. P. Feynman, R. B. Leighton, and M. Sands, The Feynman Lectures on Physics, Vol. 1 (Addison-Wesley, New York, 1963), Chap. 28.

[33] R. P. Feynman, R. B. Leighton, and M. Sands, The Feynman Lectures on Physics, Vol. 1 (Addison-Wesley, New York, 1963), Chap. 34.

[34] B. D. Patterson, A simplified approach to synchrotron radiation, Am. J. Phys. 79, 1046 (2011).

[35] A. Hofmann, The Physics of Synchrotron Radiation (Cambridge Monographs on Particle Physics, Nuclear Physics and Cosmology, Cambridge, England, 2004) Chap. 4.4.1.

[36] J. Hays-Wehle, W. Franklin, F. Kürtner, J. van der Laan, R. Milner, A. Siddiqui, C. Tschalär, and F. Wang, Interferometer design for optical stochastic cooling demonstration at Bates, Proc. SPIE, Interferometry XIV: Techniques and Analysis, 7063, 706319 (2008).

[37] R. B. Palmer, Stochastic cooling, Report No. BNL 18395, (to be published, 1973).

[38] S. Heifets and M. Zolotorev, Quantum theory of optical stochastic cooling, Phys. Rev. E 65, 016507 (2001).

[39] K.-J. Kim, Analysis of optical stochastic cooling including transverse effects, in Proc. 16th Particle Accelerator Conference, Dallas, 1-5 May 1995 (IEEE, New York, 1995), Vol. 4, p. 2786-2788.

[40] See, https://mad.web.cern.ch/mad/.

[41] A. Arno and et al., An Electron-Ion Collider Study, Tech. Rep. (https://wiki.bnl.gov/eic/upload/EIC.Design.Study .pdf BNL, 2019). 